\title{
Structure of domains in an exchange-bias model
}

\author{
A. Misraa) \\ Institut für Physik, Universität Duisburg-Essen, 47048 Duisburg, Germany and Department of Physics \\ and Astronomy and MINT Center, University of Alabama, Tuscaloosa Alabama 35487-0324 \\ U. Nowak and K. D. Usadel \\ Institut für Physik, Universität Duisburg-Essen, 47048 Duisburg, Germany
}

\begin{abstract}
The structure of domains in the interface monolayer of the antiferromagnet in an exchange-bias system is investigated in the framework of the domain state model. These interface domains carrying remanent magnetization provide the bias fiel and are strongly influence by the bulk. The stable part of the spin configuration at the interface, which is responsible for exchange bias, is identified The stability analysis of the interface domains leads to an explanation of the nontrivial dependence of the bias fiel on thickness and anisotropy of the antiferromagnet.
\end{abstract}

\section{INTRODUCTION}

The shift of the hysteresis loop along the fiel axis in a system containing a ferromagnet (FM)-antiferromagnet (AFM) interface is known as exchange bias (EB). The discovery of this effect by Meiklejohn and Bean in Co-CoO systems ${ }^{1}$ dates back to 1956 . Subsequently, the effect was observed in a variety of different materials (see Ref. 2 for a review). There have been several approaches toward a theoretical understanding of the phenomenon, and numerous different mechanisms were believed to be responsible for the shift. Malozemoff ${ }^{3}$ explained the shift as a result of interface roughness. Koon considered a spin-flo coupling between the FM and the compensated AFM interface as responsible for EB. ${ }^{4}$ However, Schulthess and Butler argued that the spin-flo coupling alone cannot account for the effect, ${ }^{5}$ but that an interface coupling through uncompensated defects together with the perpendicular coupling could be the mechanism leading to EB. ${ }^{6}$

Recently a model was proposed ${ }^{7}$ in which the cause of the shift was attributed to the extra exchange fiel provided by the irreversible magnetization of a domain state in an AFM. Here, the AFM was diluted by replacing its magnetic atoms partly by nonmagnetic impurities, and the influenc of the dilution was studied experimentally as well as in simulations. The essential idea behind the model is that when the diluted AFM is cooled below the Néel temperature in presence of the exchange fiel of the FM, it ends up in a domain state, as opposed to long-range order. The domains are metastable since the domain walls are pinned at the defects and thermal relaxation is slow at low temperatures. These domains carry a remanent magnetization ${ }^{8,9}$ that provides the biasing fiel to the FM, causing the shift of the hysteresis loop. Several issues related to EB, such as the dependence of EB on dilution, ${ }^{7}$ the role of the AFM thickness, ${ }^{10,11}$ temperature dependence, and training effect ${ }^{12,13}$ have been successfully discussed within the framework of this domain state

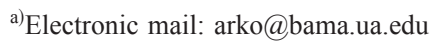

model. Support for the relevance of domains in EB systems is also given experimentally by a direct spectroscopic observation. $^{14,15}$

The importance of defects for the EB effect was also underlined by recent experiments on $\mathrm{Fe}_{x} \mathrm{Zn}_{1-x} \mathrm{~F}_{2} / \mathrm{Co}$ bilayers ${ }^{16}$ and by other experiments, ${ }^{17,18}$ which showed that it is possible to modify EB by means of irradiating an $\mathrm{FeNi} /$ FeMn system with $\mathrm{He}$ ions in presence of a magnetic field Depending on the dose of the irradiation and the magnetic fiel present at the time of irradiation, it was possible to manipulate both the magnitude and even the direction of the EB field

In the present article, a detailed study of the structure and the stability of the domains in the interface layer is presented in order to understand their role for EB. In particular, we are going to analyze how these domains respond to a change of the thickness, the anisotropy, or the dilution of the AFM, which is necessary for an understanding of the complex behavior observed in EB systems.

\section{MODEL AND METHODS}

The system we will study consists of one monolayer of FM and $t$ monolayers of an AFM. A randomly chosen fraction $p$ of AFM sites is left without a spin. The FM is exchange coupled to the topmost layer of the AFM. The Hamiltonian is given by ${ }^{19}$

$$
\begin{aligned}
\mathcal{H}= & -J_{\mathrm{FM}} \sum_{\langle i, j\rangle} \mathbf{S}_{i} \cdot \mathbf{S}_{j}-\sum_{i}\left(d_{z} S_{i z}^{2}+d_{x} S_{i x}^{2}+\mathbf{S}_{i} \cdot \mathbf{B}\right) \\
& -J_{\mathrm{AFM}} \sum_{\langle i, j\rangle} \epsilon_{i} \epsilon_{j} \boldsymbol{\sigma}_{i} \cdot \boldsymbol{\sigma}_{j}-\sum_{i} \epsilon_{i}\left(k_{z} \sigma_{i z}^{2}+\boldsymbol{\sigma}_{i} \cdot \mathbf{B}\right) \\
& -J_{\mathrm{INT}} \sum_{\langle i, j\rangle} \epsilon_{j} \mathbf{S}_{i} \cdot \boldsymbol{\sigma}_{j},
\end{aligned}
$$

where $\mathbf{S}_{i}$ and $\boldsymbol{\sigma}_{i}$ denote classical spin vectors at the $i$ th site of the FM and AFM, respectively. The firs line of the Hamiltonian describes the contribution of the FM with the $z$ axis as its easy axis with an anisotropy constant $d_{z}>0$, and the $x$ 
axis as its hard axis with an anisotropy constant $d_{x}<0$. The resulting in-plane anisotropy keeps the FM preferentially in the $y-z$ plane. The second line is the contribution from the AFM with quenched disorder $\left(\epsilon_{i}=0,1\right)$ also having its easy axis along $z\left(k_{z}>0\right)$. The last term describes the interaction of the FM with the interface AFM monolayer.

We consider nearest-neighbor interactions on a simple cubic lattice with exchange constants $J_{\mathrm{FM}}$ and $J_{\mathrm{AFM}}$ for the FM and the AFM, respectively, while $J_{\text {INT }}$ denotes the exchange constant between the FM and the AFM. The exchange constants for the FM and the AFM mainly determine their respective ordering temperatures; in our simulations, we set $J_{\mathrm{FM}}=-2 J_{\mathrm{AFM}}$, so that the Curie temperature is well above the Néel temperature. The strength or even the sign of the interface exchange interaction are usually not known from experiments. We set $J_{\mathrm{INT}}=J_{\mathrm{FM}} / 2$, as was done in our previous work, since changing this parameter changes the results only quantitatively. The entire system is placed in an external magnetic fiel $\mathbf{B}=B \hat{z}$. In the following, field are measured in terms of $J_{\mathrm{FM}}$ and all spin vectors are normalized to unity.

We use Monte Carlo methods with a heat-bath algorithm and single-spin-fli dynamics for the simulations. At every Monte Carlo step, each spin is subjected to a trial step consisting of a small deviation from the original direction followed by a total flip This twofold trial step can take care of a broad range of anisotropies starting from very soft spins up to the Ising limit. ${ }^{20}$ The lateral dimension of the system is chosen in such a way that multiple AFM domains can be observed, which is the case for a lattice size of $128 \times 128$ $\times(t+1)$, where $t$ is the thickness of the AFM. We use periodic boundary condition along the lateral directions of the system and open boundary condition in the direction perpendicular to the film Unless specificall mentioned otherwise, all the results presented in the following are obtained for an AFM dilution of $p=0.4$. Starting from a temperature above the Néel temperature $T_{\mathrm{N}}(p)$ of the diluted AFM but below the Curie temperature $T_{\mathrm{C}}$ of the FM, the system is cooled below $T_{\mathrm{N}}$ in presence of an external magnetic fiel $\mathbf{B}$ $=0.25 J_{\mathrm{FM}} \hat{z}$. The fina temperature is $T=0.1 J_{\mathrm{FM}}$, at which the hysteresis curve is simulated. The EB fiel is determined as $B_{\mathrm{EB}}=\left(B^{+}+B^{-}\right) / 2$, where $B^{+}$and $B^{-}$are those values of the fiel at which the $z$ component of the FM magnetization is zero when the fiel is decreased and increased, respectively.

\section{RESULTS}

\section{A. AFM domains and their structure}

Formation of domains in the AFM is an essential preposition for the investigation of EB in the framework of the domain state model. During cooling the AFM in the presence of an external fiel through its Néel temperature, a domain state is formed within the AFM. A discussion about the formation of these domains and the reason for their stability was provided before. ${ }^{12,19}$ These domains penetrate the AFM layer. In the following, we will provide a detailed study of the stability and the structure of these domains, especially at

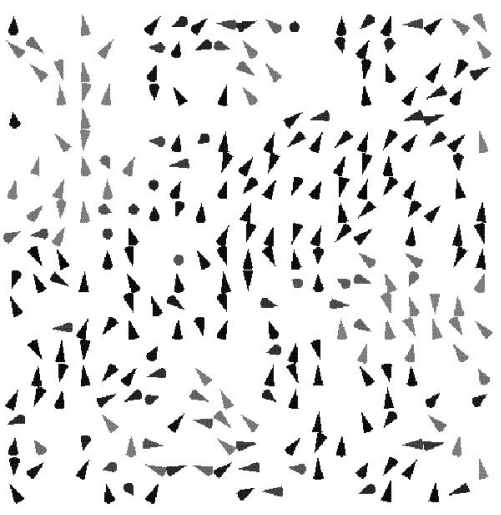

(a)

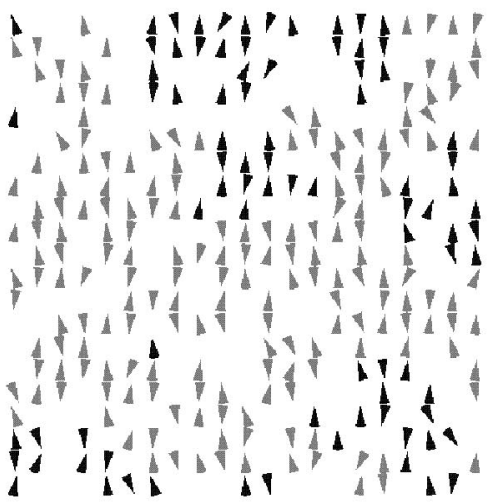

(b)

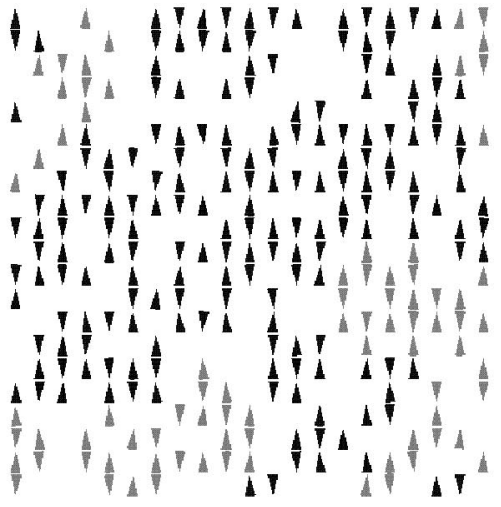

(c)

FIG. 1. Typical snapshots of spin configuration in a small portion of the interface monolayer of the AFM showing the domain structure for AFM anisotropies: (a) $k_{z}=0.1 J_{\mathrm{FM}}$, (b) $k_{z}=J_{\mathrm{FM}}$, and (c) $k_{z}=30 J_{\mathrm{FM}}$. The thickness of the AFM is $t=3$. The color coding distinguishes different AFM domains.

the interface because only those spins have a direct influenc on the FM through the nearest neighbor exchange coupling.

Typical snapshots of "frozen" AFM interface domains, obtained immediately after fiel cooling, are shown in Fig. 1. As can be seen clearly the domain walls are wider for smaller values of $k_{z}$, whereas Ising-like domain structures are obtained for stronger anisotropies. Snapshots showing how such domains in the AFM evolve when going away 


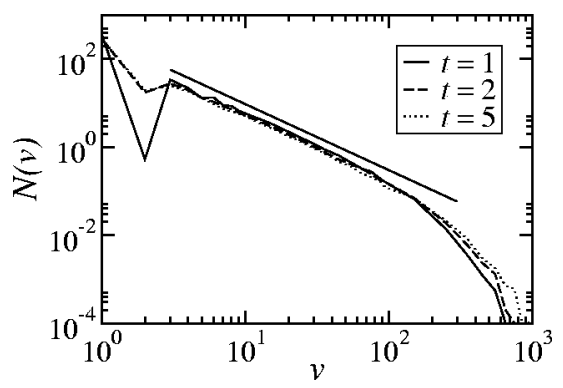

FIG. 2. Power law distribution of the size of the AFM interface domains for different AFM thicknesses. The data have been logarithmically averaged. The reference straight line has a slope of -1.5 .

from the interface have already been shown before. ${ }^{19}$ In addition, for the Ising case, it was reported earlier ${ }^{10}$ that the size of the interface domains increases with increasing $t$. The present simulation shows that this behavior is not unique to Ising spins, but is also observed for softer spins.

Figure 2 depicts the size distribution of the interface domains for $k_{z}=J_{\mathrm{FM}}$, also showing how the distribution changes with $t$. The size of a domain is define as the number of spins it consists of. The distribution is averaged over different domain configuration obtained upon fiel cooling of the sample. The mean number of domains $N$ for a given size $v$ follows a power law with an exponential cutoff:

$$
N(v)=N_{0} v^{-d} e^{-v / v_{0}} .
$$

The cutoff parameter $v_{0}$ depends on $t$. A similar behavior was observed earlier for pure diluted AFMs in a field where the cutoff varied with the applied field ${ }^{21}$ The reference straight line in Fig. 2 has a slope of $d=1.5$, the same value as reported in Ref. 21. When there is only one monolayer of the AFM in contact with the FM, in a domain with $v=2$ one of the spins is frustrated. Therefore, such domains are not stable even if the domain boundaries pass exclusively through impurities. This is the reason for the sharp kink at $v=2$ in the graph corresponding to $t=1$. This kink is drastically reduced upon addition of one more monolayer, which reduces the frustration. This fact is also reflecte in the distribution of the normalized domain magnetization $m_{d}$ shown in Fig. 3. Here, the distribution shows the mean number of domains $N$ for a given value of $m_{d}$ and the peaks in the distribution correspond to different domain sizes. Note that the peak at $m_{d}$ $=0$ for the curve corresponding to $t=1$ is extremely small

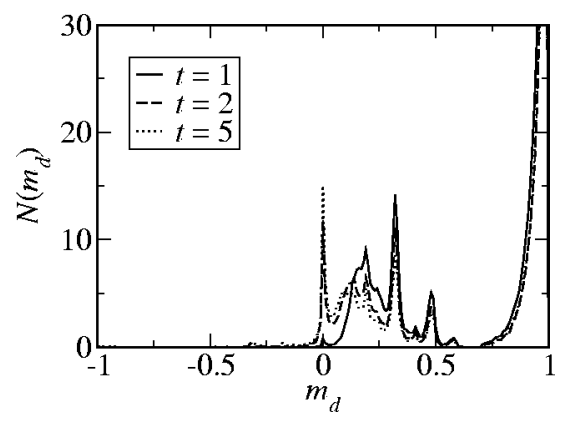

FIG. 3. Distribution of the normalized domain magnetization $m_{d}$ for different AFM thicknesses.

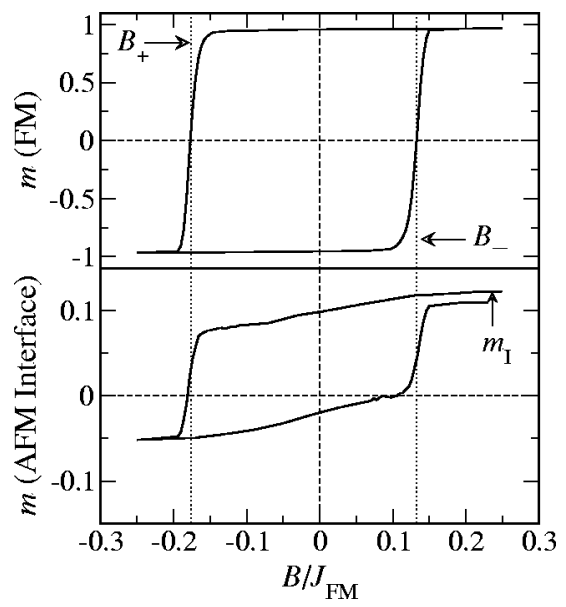

FIG. 4. Typical simulated hysteresis loops for the FM (a) and interface monolayer of the AFM (b).

since, here, primarily domains with $v=2$ contribute. The width of the peaks is directly related to the anisotropy of the AFM tending to zero in the Ising limit.

The fact that $v_{0}$ increases with $t$ in Fig. 2 indicates that bigger interface domain sizes are preferred for a thicker AFM. The reason for such a behavior has been indicated before. As more monolayers of AFM are added, it requires more energy for a formation of perpendicular domain walls. The system responds by reducing the number of smaller domains and increasing the size of the bigger domains (see also Fig. 2 in Ref. 10). In this way, the domain structure becomes coarser, that is, with less smaller domains and smoother domain walls. This leads to the downward shift of the curve corresponding to higher $t$ values in Fig. 2 and to larger $v_{0}$ for increasing $t$.

\section{B. Hysteresis loops}

A typical hysteresis loop corresponding exclusively to the FM part of the system is shown in the top part of Fig. 4. The hysteresis loop at the bottom corresponds to the interface monolayer of the AFM. The existence of nonzero loop area of the latter proves the existence of remanent magnetization in the AFM domains. The most important feature of this loop is its rather strong upward shift. It shows that part of the magnetization in the AFM picked up during cooling is frozen; that is, it cannot follow the FM during fiel reversal. On the other hand, those spins which switch reversibly during the cycle give rise to the nonzero area of the hysteresis loop.

The shift of the loop is always upward for positive $J_{\text {INT }}$ because the "frozen domains" carry a positive net magnetization as a consequence of cooling the system in presence of a positive external fiel and a positive exchange fiel due to the FM. The effect of these frozen AFM spin configuration is that they carry an irreversible domain state magnetization, which provides the extra positive fiel acting on the FM which requires a stronger coercive fiel during the forward cycle as compared to the coercive fiel during the return cycle of the hysteresis curve. EB, which is the result of such an asymmetry, is therefore directly related to this upward 


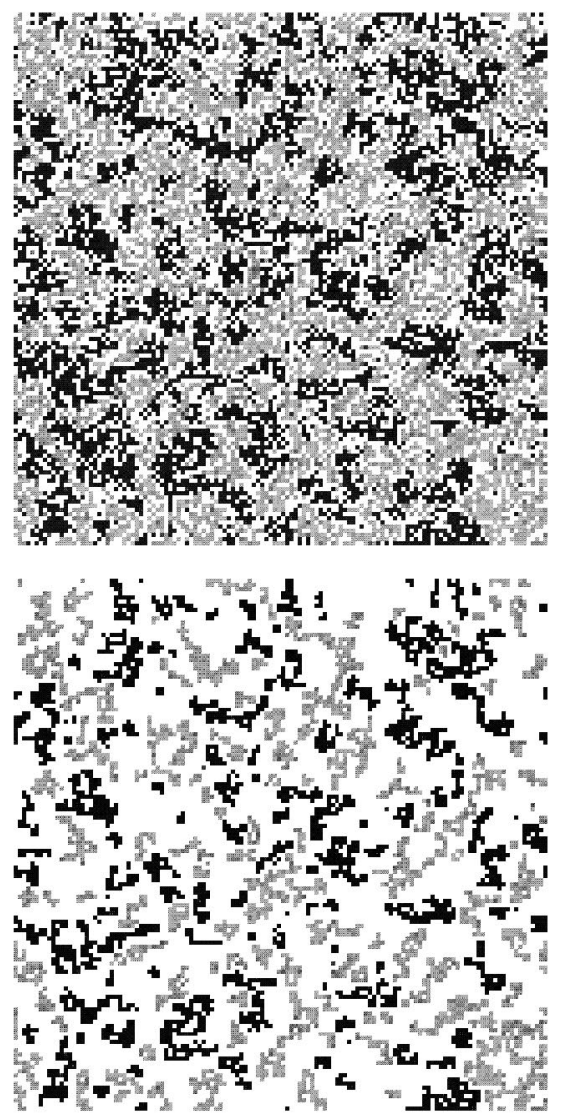

FIG. 5. Staggered spin configuratio of the interface monolayer for an AFM thickness of $t=1$. The top snapshot shows the initial spin configuratio after fiel cooling, while the bottom snapshot shows the stable part of it.

shift of the AFM interface hysteresis loop. Further implications of this upward shift are discussed later in the article.

\section{Stability of the interface}

Obviously, the AFM interface, which is exchange coupled to the FM, is required to carry nonzero remanent magnetization along the $z$ direction to give rise to EB. In addition, this remanent magnetization must be partly stable during a complete hysteresis cycle.

A way of measuring the stability of an individual spin is to look at a time average of its $z$ component during a complete hysteresis loop. This average value will be close to its initial value for a stable spin, whereas it tends to zero if the spin follows the external field A quantitative measure for the stability of an individual spin is the ratio of this average divided by the initial spin value. A suitably chosen cutoff value for this ratio separates the stable spins from the unstable ones.

Figure 5 shows typical staggered configuration of stable spins at the AFM interface monolayer for $t=1$. The upper snapshot shows the initial spin configuratio immediately after fiel cooling and the lower one shows the corresponding stable part of it. For $t=1$, the domain walls can move to a certain extent while the FM is reversed. Therefore, only those spins which are far away from the domain boundary are stable. Figure 6 shows the dependence of the stable spin
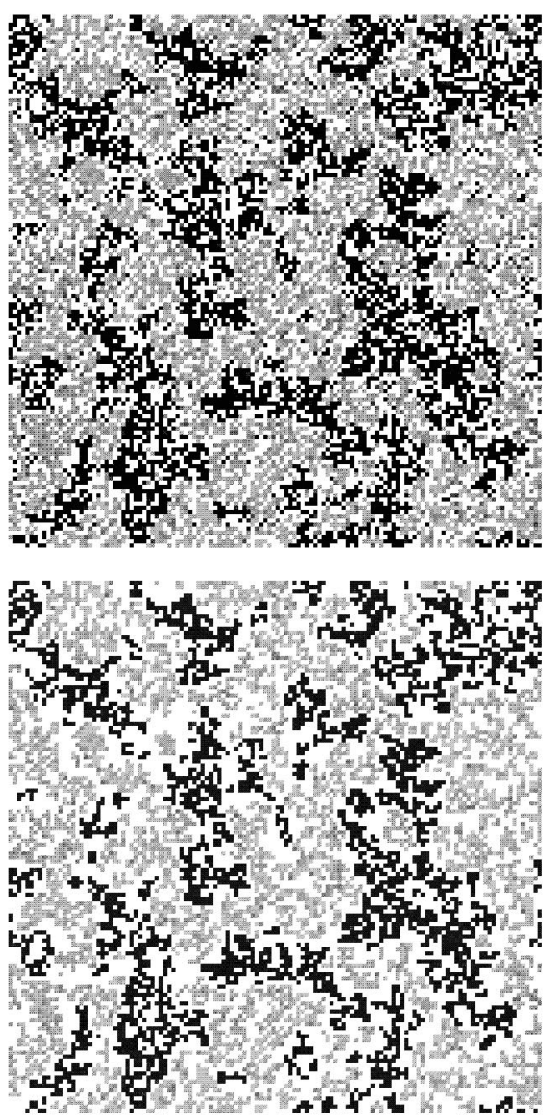

FIG. 6. Staggered spin configuratio of the interface monolayer for an AFM thickness of $t=5$. The top snapshot shows the initial spin configuratio after fiel cooling, while the bottom snapshot shows the stable part of it.

configuration on AFM thickness. The domain walls for this case are now less free to move since they are attached to the spins in the next AFM layer leading to a higher amount of stable spins at the interface.

We defin the stability $\alpha$ of the whole AFM interface as the average value of the stability of individual spins at the interface. Figure 7 shows how $\alpha$ increases with the thickness of the AFM for different anisotropies. The curves shift upward with increasing anisotropy and those corresponding to $k_{z}=J_{\mathrm{FM}}$ and $30 J_{\mathrm{FM}}$ show a sharp rise of the stability from $t=1$ to $t=2$, whereupon it reaches its saturation value. For only one monolayer of AFM, its interface interaction with the FM layer which follows the external fiel destabilizes the

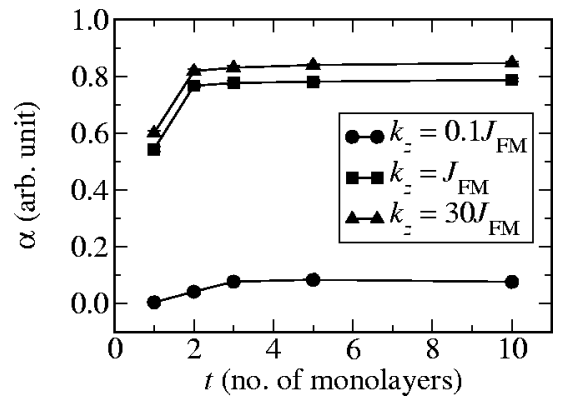

FIG. 7. Stability of the interface AFM as a function of thickness of the AFM. 


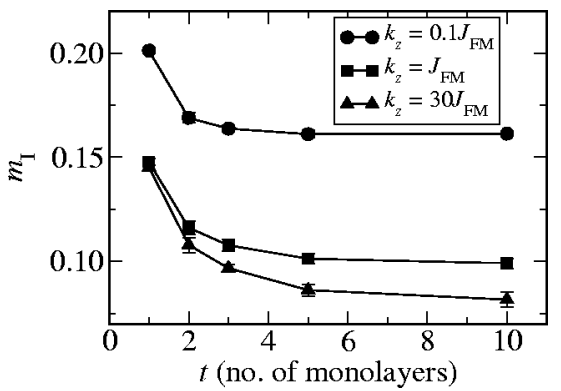

FIG. 8. Influenc of the thickness on the normalized interface magnetization $m_{\mathrm{I}}$ of the AFM.

AFM. Upon addition of only one more monolayer, this effect is balanced by the additional exchange interaction with the second AFM monolayer. This increases the stability of the interface monolayer quite drastically. However, at very low anisotropies the stability of the interface is extremely low because the spins are more free to move away from their easy axis becoming more aligned with the FM. This is the reason the stability for $k_{z}=0.1 J_{\mathrm{FM}}$ continues to increase for higher values of thickness as compared to its behavior for higher values of $k_{z}$.

\section{Interface magnetization}

As was already shown in Fig. 3, the AFM interface contains nonzero magnetization after fiel cooling. We defin $m_{\mathrm{I}}$ as the net magnetization of the AFM interface. This quantity is the area under the curve in Fig. 3; we have also indicated this quantity in the AFM hysteresis loop in Fig. 4. Figure 8 shows the dependence of $m_{\mathrm{I}}$ on the thickness of the AFM for different values of the anisotropy. In all cases, the interface magnetization has its maximum value for $t=1$, and it gradually decreases with increasing thickness before reaching a saturation value for large $t$.

The reason for this decrease of the interface magnetization is the change of the domain structure, as discussed in Sec. A. With increasing AFM thickness, the domain structure becomes coarser with smoother domain walls. This reduces the magnetization that is stored in the domain structure. The downward shift of the curves with increasing anisotropy is due to a more effective cancellation among oppositely oriented neighboring spins in a domain.

\section{E. Role of AFM thickness for EB}

In an earlier communication, ${ }^{10}$ we reported on the dependence of the bias fiel on AFM thickness $t$. It was found that the absolute value of bias fiel firs increases with the thickness up to a maximum and then decreases again, attaining a saturation value. In that report, a strong AFM anisotropy was assumed; that is, the AFM was treated as Ising model. However, this nontrivial behavior of the bias fiel with varying AFM thickness is not restricted to the Ising limit of the AFM. Figure 9(a) shows data for finit values of $k_{z}$. The highest value for the anisotropy used is $k_{z}$ $=30 J_{\mathrm{FM}}$, which corresponds to the Ising limit. Except for very low AFM anisotropy, the absolute value of the bias fiel has a pronounced peak at a thickness of $t=2$. The height of
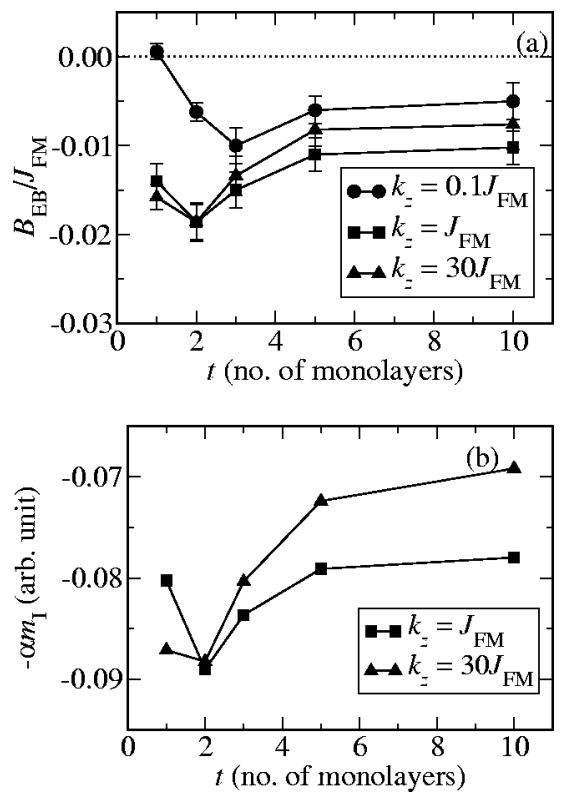

FIG. 9. (a) Variation of the EB fiel with the thickness of the AFM for different AFM anisotropies $k_{z}$. (b) Qualitative reproduction of the effect of AFM thickness on the EB field The relative position of the two curves and the position of their peaks is identical to that in (a). An arbitrary scale is used along the ordinate.

the peak, however, increases with decreasing value of $k_{z}$, as long as $k_{z}$ is not too small. The position of the peak is shifted to higher values for $k_{z}=0.1 J_{\mathrm{FM}}$ This nontrivial dependence of EB on the AFM thickness was already observed in several experiments (see Refs. 2, 13 and references therein).

The reason for this nontrivial dependence is a competition between the stability of the AFM interface with increasing AFM thickness and the strength of AFM interface magnetization $m_{\mathrm{I}}$. Indeed, if we multiply the stability factor $\alpha$ with $m_{\mathrm{I}}$, the result obtained [as shown in Fig. 9(b)] is qualitatively similar to that in Fig. 9(a). It is important to note how the behavior of the bias fiel at the two extreme values of $t$ is correctly reproduced in the figure For small values of $t, k_{z}$ is solely responsible for the stability of the interface AFM domains. Therefore, for $t \leqslant 2$, the bias is stronger for stronger anisotropies. The situation reverses upon addition of more layers since then the bulk is predominantly providing the stability. However, since the value of $m_{\mathrm{I}}$ decreases with increasing $k_{z}$, the bias fiel becomes stronger for lower anisotropy. The appearance of the peak at $t=2$ can also be explained by noting the sharp increase in $\alpha$ from $t=1$ to $t$ $=2$ followed by a moderate increase for higher values of $t$. Since $m_{\mathrm{I}}$ decreases gradually with increasing $t$, the product of $\alpha$ and $m_{\mathrm{I}}$ shows a peak at $t=2$. As we mentioned before, for very low anisotropies it is quite difficul to differentiate between the stable and the unstable spins, so that the behavior of the bias fiel is not very well reproduced from the stability analysis in this limit.

\section{F. Coercivity}

Another quantity of interest in EB systems is the coercivity. The coercive fiel for the FM is define as $B_{\mathrm{CO}}$ $=\left(B^{-}-B^{+}\right) / 2$. The sharp rise and fall of the magnetization 


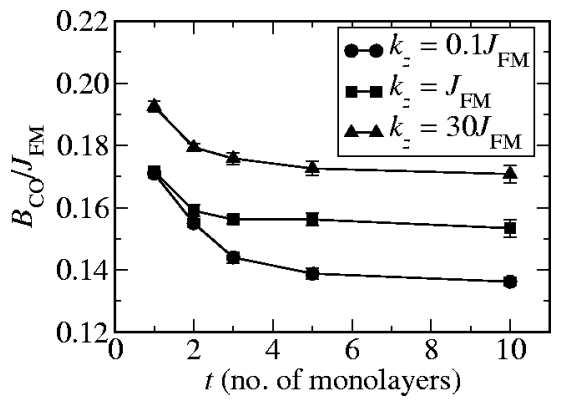

FIG. 10. Influenc of thickness and anisotropy of the AFM on coercivity $B_{\mathrm{CO}}$.

along the increasing and decreasing fiel branch of the loop seen in the upper part of Fig. 4 results from the anisotropy of the FM.

However, the strength of the coercive field is influence by the AFM and therefore also depends on the thickness and the anisotropy of the AFM, as can be seen in Fig. 10. The decreasing value of $B_{\mathrm{CO}}$ with increasing thickness can be understood as follows. The interface magnetization $m_{\mathrm{I}}$ of the AFM tries to orient the FM along its direction. The coercive fiel has to overcome this barrier, and the higher the interface magnetization of the AFM the stronger is the fiel required to reverse the FM. Since the interface magnetization decreases with increasing thickness, as is already depicted in Fig. $8, B_{\mathrm{CO}}$ behaves in a similar fashion. However, $k_{z}$ also has a substantial effect on the coercive field It requires a stronger external field to rotate a FM spin which is connected to a "harder" AFM spin. Therefore, the curves in Fig. 10 shift upward as $k_{z}$ is increased.

\section{G. Role of AFM anisotropy on EB}

We have calculated the bias fiel for a wide range of values of $k_{z}$, starting from very soft spins to rigid, Ising-like spins. Figure 11 shows result for different thicknesses of the AFM for two values of the dilution: $p=0.4$ and 0.6. A logarithmic scale is used along the $k_{z}$ axis only to increase the clarity of the figures In the upper part of Fig. 11(a) it is shown that for $p=0.4$ a thick AFM produces a maximum in the absolute value of the EB fiel at an intermediate value of $k_{z} \approx J_{\mathrm{FM}}$, while at lower thicknesses of the $\mathrm{AFM}$, the $\mathrm{EB}$ fiel increases with the anisotropy and saturates in the Ising limit.

This behavior can be understood with the help of the stability criterion of the interface AFM. The stability of the interface spins, at a particular thickness, increases with increasing anisotropy, as shown in Fig. 7. Therefore, one would expect the EB to increase monotonically with $k_{z}$. This happens at very low thickness of the AFM where, in absence of the bulk, "stiffness" of the spins is the sole parameter responsible for the stability. However, at higher values of $t$, the stability is predominantly provided by the thickness and, hence, the effect of AFM anisotropy becomes less signifi cant. As shown in Fig. 8, the net magnetization $m_{\mathrm{I}}$ contained in the interface upon fiel cooling decreases with increasing anisotropy. This happens because for higher values of $k_{z}$ the domain walls cost more energy and the system responds by
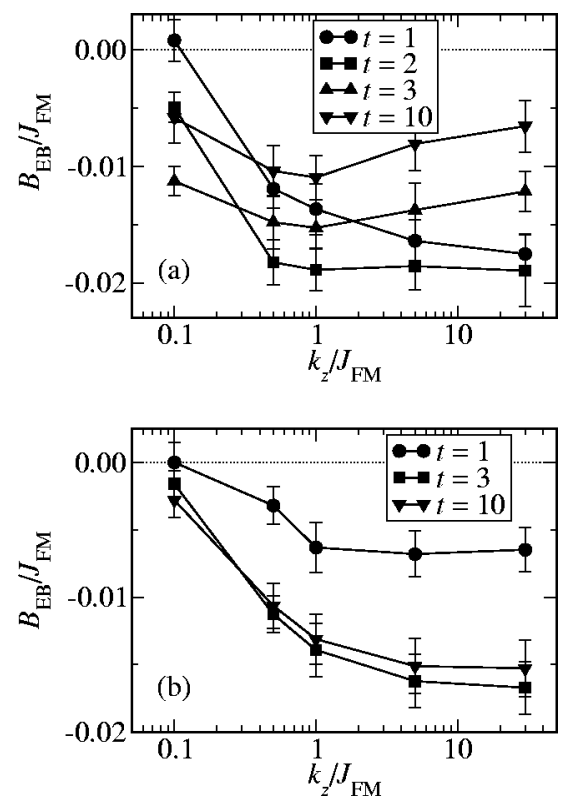

FIG. 11. Bias fiel versus the AFM anisotropy for different values of thickness $t$ : (a) $p=0.4$ and (b) $p=0.6 . k_{z}$ is measured in units of $J_{\mathrm{FM}}$.

making the domain walls flatte. This in turn reduces the remanent magnetization of the domains. Therefore at higher values of $t$, the bias fiel increases initially with $k_{z}$ to a maximum before it decreases again at higher values of $k_{z}$.

The situation changes for higher dilution. As can be seen in the lower part of Fig. 11, the peak in the bias fiel disappears for all values of $t$. This happens when we are close to the percolation threshold, at which the domain walls pass nearly exclusively through the defects costing very little or no energy. Since no smoothening of the domain walls is now required to minimize the energy for domain formation, the remanent magnetization at the interface does not change significantl with increasing thickness. Hence, EB increases with $k_{z}$ until it saturates in the Ising limit.

\section{CONCLUSIONS}

In this article, we have studied the structure of the interface domains of the AFM and their response to a change in the thickness and the anisotropy of the AFM in an EB system. The size distribution of the interface domains follows a power law with an exponential cutoff. The power law exponent was found to be 1.5 , in agreement with the value obtained for a pure diluted AFM in a field ${ }^{21}$ The cutoff value increases with the thickness of the AFM, implying that bigger domains are formed for larger thicknesses, a result firs observed in an EB system with an Ising AFM. ${ }^{10}$

We have shown that there are two quantities that are crucial for understanding the strength of the EB field These are the interface magnetization of the AFM after fiel cooling on the one hand, and its stability over a complete hysteresis loop on the other hand. The product of these two quantities is the irreversible domain state magnetization, which is identifie as the amount of the upward shift of the hysteresis loop of the AFM interface. It explains the strength of the EB 
quantitatively. The behavior of the coercive fiel can also be understood with this framework.

Both the anisotropy of the AFM and its thickness play a very important and highly complicated role in determining the value of this bias field The dependence of EB on $k_{z}$ is also nontrivial. Instead of a monotonic increase of the bias fiel with $k_{z}$, which one might naively expect, a peak in the absolute value of the bias fiel at some intermediate value of the AFM anisotropy is obtained. However, this peak disappears at a lower thickness or at a dilution close to the percolation threshold.

It has widely been understood that not all the spins in the AFM interface are responsible for the EB effect and we have clearly identifie those spins that are frozen in time and, hence, are responsible for the EB. Most importantly, we would like to stress that even though EB is predominantly an interface effect, the bulk plays a significan role by means of influencin the domain structure of the AFM interface.

\section{ACKNOWLEDGMENTS}

This work was supported by the Deutsche Forschungsgemeinschaft through Graduiertenkolleg 277 and SFB 491.

${ }^{1}$ W. H. Meiklejohn and C. P. Bean, Phys. Rev. 102, 1413 (1956).

${ }^{2}$ J. Nogués and I. K. Schuller, J. Magn. Magn. Mater. 192, 203 (1999).

${ }^{3}$ A. P. Malozemoff, Phys. Rev. B 37, 7673 (1988).
${ }^{4}$ N. C. Koon, Phys. Rev. Lett. 78, 4865 (1998).

${ }^{5}$ T. C. Schulthess and W. H. Butler, Phys. Rev. Lett. 81, 4516 (1998).

${ }^{6}$ T. C. Schulthess and W. H. Butler, J. Appl. Phys. 85, 5510 (1999).

${ }^{7}$ P. Miltényi, M. Gierlings, J. Keller, B. Beschoten, G. Güntherodt, U. Nowak, and K. D. Usadel, Phys. Rev. Lett. 84, 4224 (2000).

${ }^{8}$ W. Kleemann, Int. J. Mod. Phys. B 7, 2469 (1993).

${ }^{9}$ D. P. Belanger, in Spin Glasses and Random Fields, edited by A. P. Young (World Scientific Singapore, 1998).

${ }^{10}$ U. Nowak, A. Misra, and K. D. Usadel, J. Appl. Phys. 89, 7269 (2001).

${ }^{11}$ M. S. Lund, W. A. A. Macedo, K. Liu, J. Nogués, I. K. Schuller, and C. Leighton, Phys. Rev. B 66, 054422 (2002).

${ }^{12}$ U. Nowak, K. D. Usadel, P. Miltényi, J. Keller, B. Beschoten, and G. Güntherodt, Phys. Rev. B 66, 14430 (2002).

${ }^{13}$ J. Keller, P. Miltényi, B. Beschoten, G. Güntherodt, U. Nowak, and K. D. Usadel, Phys. Rev. B 66, 14431 (2002).

${ }^{14}$ F. Nolting, A. Scholl, J. Stöhr, J. W. Seo, J. Fompeyrine, H. Siegwart, J.-P. Locquet, S. Anders, J. Lüning, E. E. Fullerton, M. F. Toney, M. R. Scheinfein, and H. A. Padmore, Nature (London) 405, 767 (2000).

${ }^{15}$ H. Ohldag, A. Scholl, F. Nolting, S. Anders, F. U. Hillebrecht, and J. Stöhr, Phys. Rev. Lett. 86, 2878 (2001).

${ }^{16}$ H. T. Shi, D. Lederman, and E. E. C. Fullerton, J. Appl. Phys. 91, 7763 (2002).

${ }^{17}$ T. Mewes, R. Lopusnik, J. Fassbender, B. Hillebrands, M. Jung, D. Engel, A. Ehresmann, and H. Schmoranzer, Appl. Phys. Lett. 76, 1057 (2000).

${ }^{18}$ A. Mougin, T. Mewes, M. Jung, D. Engel, A. Ehresmann, H. Schmoranzer, J. Fassbender, and B. Hillebrands, Phys. Rev. B 63, 60409 (2001).

${ }^{19}$ U. Nowak, A. Misra, and K. D. Usadel, J. Magn. Magn. Mater. 240, 243 (2002).

${ }^{20}$ U. Nowak, in Annual Reviews of Computational Physics IX, edited by D. Stauffer (World Scientific Singapore, 2000), p. 105.

${ }^{21}$ U. Nowak, J. Esser, and K. D. Usadel, Physica A 232, 40 (1996). 